\title{
Abordagem Semiótica à Inspeção de Interfaces: Um estudo de caso da plataforma TIM Tec
}

Semiotic Approach to Interface Inspection: A TIM Tec platform casa study.

KIANE, Rayse; mestranda; Universidade Federal de Santa Catarina

raysekiane@gmail.com

PERASSI, Richard; Professor Dr.; Universidade Federal de Santa Catarina

richard.perassi@uol.com.br

\section{Resumo}

Este trabalho realiza um estudo de caso na interface gráfica de uma vídeo-aula do sistema MOOC TIM Tec, através de uma inspeção semiótica utilizando uma tipologia para signos computacionais e um framework para a interpretabilidade dos mesmos. O resultado final apresenta uma predominância de signos interativos, que apresentam características permanentes, de manuseio e transientes, e compostos apenas de elementos textuais que apresentam ótimo feedback para as ações dos usuários. $O$ framework se mostrou adequado para a avaliação da interpretabilidade dos signos, porém, não esgota o assunto, sendo necessária sua aplicação em outros tipos de sistemas e mais avaliações e testes com usuários para uma percepção completa da interpretabilidade do usuário.

Palavras Chave: Semiótica computacional; inspeção semiótica; experiência do usuário.

\begin{abstract}
This work is a semiotic inspection using a typology for computational signs and a framework for the interpretability of the signs. The final result presents a predominance of interactive signs, which presented characteristics, materials and transients, and composed only of textual elements that are excellent feed back for user actions. The framework was valid for an evaluation of the interpretability of the signs, however, does not exhaust the subject, being its application in other kinds of systems and evaluation and testing with users for a complete perception of user interpretability.
\end{abstract}

Keywords: Computacional semiotics; semiotic inspection; user experience. 


\section{Introdução}

Interface Gráfica do Usuário (IGU), traduzido do termo inglês Graphical User Interface (GUI), é o meio que permite a interação do usuário com dispositivos digitais através de imagens e indicadores visuais. Desde a criação do computador a IGU passou por um longo processo de evolução até chegar nos ambientes ricos em cores e ícones e interfaces sensíveis ao toque que conhecemos hoje. Inicialmente o desenvolvimento dos computadores e suas aplicações eram focados nas tecnologias e nos especialistas, físicos, matemáticos e programadores (BARANAUSKAS et al, 1998). Já nos anos de 1980 com o começo da popularização do uso do computador em diversas profissões, e se tornando objeto de uso pessoal dentro das residências, diversas empresas focaram seus esforços em tornar as máquinas mais amigáveis aos usuários, principalmente desenvolvendo a IGU.

A área de estudo da Interação Humano Computador (IHC) como definido pela Sociedade Brasileira de Computação (2018), se dedica a estudar os fenômenos de comunicação entre pessoas e sistemas computacionais e está na interseção das ciências da computação e informação, e ciências sociais e comportamentais, envolvendo todos os aspectos relacionados com a interação entre usuários e sistemas. Para Preece e Rogers (1994), interface é a parte do sistema computacional com a qual o usuário se comunica, onde ações são trocadas entre os agentes, usuário e computador, e este processo chama-se interação. Oliveira, Luz e Prates (2008) destacam a importância de métodos integrativos na área de avaliação de IHC. Desta forma, a interação do usuário com os elementos gráficos pode ser analisada sob uma perspectiva semiótica, ciência que estuda os signos e os fenômenos de produção de significação e sentido (BARANAUSKAS et al, 1998), a fim de enriquecer a avaliação e melhorar a experiência do usuário.

Para o estudo de IGU Andersen (1997) utiliza o conceito de "Semiótica Computacional", uma disciplina da semiótica que estuda a natureza e uso de signos baseados no computador, assim a semiótica deve necessariamente considerar os sistemas de informação como canais de sinais cuja principal função é ser percebida e interpretada por um grupo de usuários, não tendo nada a dizer sobre os dados em si, apenas na sua capacidade de ser interpretado e usado como fonte de conhecimento ou guia para uma ação (ANDERSEN, 1992).

Este trabalho tem como objetivo inspecionar uma interface gráfica de um sistema educacional sob a perspectiva da semiótica. Desta forma, utiliza a metodologia proposta por Baranauskas et al (1998) para o estudo de uma IGU do sistema TIM Tec, aplicando um framework teórico para signos computacionais seguindo os conceitos de Andersen (1997) e Familant e Detweiler (1993), apresentados nas seções de referencial teórico e metodologia.

\section{MOOC TIM Tec}

TIM Tec é uma iniciativa brasileira do Instituto TIM como uma ferramenta de apoio para os objetivos do PRONATEC, Programa Nacional de Acesso ao Ensino Técnico e Emprego. Segundo o site do Portal TIM Tec o programa possui o objetivo de expandir, interiorizar e democratizar a oferta presencial e à distância de cursos técnicos e de formação inicial e continuada (FIC). O TIM Tec é composto por 3 pilares, o software, os cursos e a rede. O software é livre, sob licença AGPL v3, e pode ser instalado ou modificado por qualquer instituição de ensino ou organização interessada em ter sua própria plataforma $\mathrm{MOOC}$, curso aberto online e massivo, todo código e tutorial necessário para a instalação encontra-se disponível no Github. O TIM Tec desenvolve cursos para a plataforma nas mais diversas áreas, e estão disponíveis tanto para serem cursados por qualquer pessoa quanto 
para baixar o pacote de curso e instalar em outra plataforma MOOC TIM Tec. A Rede TIM Tec é formada por todas as instituições que adotam e instalam a plataforma TIM Tec, e que utilizam seus cursos. Ela é formada majoritariamente pelas instituições de ensino da Rede e-Tec Brasil, mas também inclui instalações independentes (TIMTEC, 2018).

\section{Referencial Teórico}

A semiótica computacional não se difere dos fundamentos da semiótica americana apresentada por Charles Peirce, que é uma ciência que estuda os processos lógicos gerais. Pode ser considerada a ciência das leis gerais dos signos (PEIRCE, 1994). Computadores em toda a sua essência são máquinas simbólicas construídas e controladas por meio de signos, talvez não explicitamente ao usuário que só percebe o lado gráfico digital deste aspecto.

Nadin (1988) afirma que a quantidade de signos na interface influencia o tempo requerido para processamento humano, enquanto que o tipo de signo afeta as espécies de processos envolvidos. Para fazer a inspeção de interface este estudo de caso se baseia nos procedimentos utilizados por Baranauskas et al (1998), primeiramente utilizando a tipologia de signos baseados no computador de Andersen (1997) e posteriormente utilizando o framework de Familant e Detweiler (1993) que permite certo grau de inferência sobre a interpretabilidade dos signos na interface.

\subsection{Tipologia dos Signos Computacionais}

Para Andersen (1992), os signos computacionais são compostos por três características de recursos, são elas:

- Manuseio: signo produzido pelo usuário ou que permite uma ação deste sobre si e inclui movimentos de tecla, mouse e joystick, e esta ação tem algum significado para a interface.

- Permanentes: característica gerada pelo computador. É uma propriedade do signo que permanece constante ao longo de toda a vida de um signo.

- Transientes: gerada pelo computador, mas ao contrário das características permanentes, ela muda à medida que o signo é usado. Não contrasta principalmente com outros signos, mas apenas internamente no mesmo signo, simbolizando os diferentes estados nos quais o signo referente pode estar.

Por exemplo, o botão de curtir da rede social Facebook apresenta as três características de recursos, podendo ser pressionado (característica de manuseio), possui forma e texto explicativo fixos (característica permanente) e apresenta cores diferentes de acordo com seu estado, pressionado ou não (característica transiente).

Figura 1 - Botão com as três características de recurso.

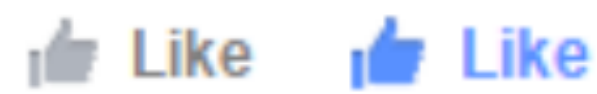

Fonte: Facebook.com (2018), adaptado pelo autor.

Com estas características Andersen (1992) sugere uma tipologia, apresentada na figura 2, 
compostas de signos dos tipos:

Figura 2 - Tipologia dos signos computacionais.

\begin{tabular}{|c|l|l|l|l|}
\cline { 3 - 4 } \multicolumn{2}{c|}{} & \multicolumn{2}{c|}{ +ação } & \multirow{2}{*}{-ação } \\
\cline { 3 - 4 } \multicolumn{2}{c|}{} & +manipulação & -manipulação & \\
\hline \multirow{2}{*}{+ permanente } & +transiente & Interativo & Ator & Objeto \\
\cline { 2 - 4 } & -transiente & Botão & Controlador & Layout \\
\hline -permanente & & \multicolumn{2}{|c|}{ Fantasma } \\
\hline
\end{tabular}

Fonte: Andersen (1992).

- Interativo: Manipulados diretamente pelo usuário, disparam uma ação em resposta, por exemplo um botão de iniciar um programa.

- Atores: Podem modificar sua posição e/ou forma na tela e influenciar outros signos, mas não podem ser influenciados diretamente pelo usuário, por exemplo barras indicativas de progresso.

- Controladores: Mudam propriedades de outros signos, mas não mudam as suas próprias, por exemplo bordas de janelas, quando atinge as bordas, o cursor tem sua forma alterada.

- Objetos: possuem aspectos transientes e permanentes, mas não aspectos de manuseio, por exemplo um texto em um editor de texto pode ter suas características alteradas (fonte, cor), mas não dispara ações que se refletem em outros signos.

- Botões: assemelham-se aos Interativos, apenas as suas características transientes são rudimentares, por exemplo ao invés de disparar uma ação este muda de cor ou ganha algum destaque.

- Layout: não possuem aspectos transientes e de manuseio. Servem como decoração, uma vez que só possuem aspectos permanentes. Ex: Figura de fundo na tela.

- Fantasma: não possuem aspectos permanentes, transientes e de manipulação. Ex: armadilhas invisíveis em jogos de videogame.

\subsection{Interpretabilidade dos Signos}

Familant e Detweiler (1993), afirmam que para comunicar a informação de forma eficaz, ela deve ser codificada fisicamente primeiro como um "sinal", isto é, informação comunicativa no signo (BARANAUSKAS et al, 1998), no caso de sistemas computacionais estes sinais são interpretados como os elementos gráficos das IGU. Para Familant e Detweiler (1993) um signo é uma relação entre o sinal e o referente, para um intérprete. Por exemplo, o pincel em um software de desenho, que tem como "sinal" é o conjunto de pixels na tela e o "referente" no mundo real, o pincel propriamente dito (BARANAUSKAS et al, 1998). Esta relação sígnica, entre o sinal e o referente, pode ser entendida como icônica ou simbólica. Relações icônicas são aquelas em que as propriedades do sinal são comuns as propriedades do referente, já nas relações simbólicas qualquer similaridade entre o sinal e seu referente é acidental (FAMILANT e DETWEILER, 1993; BARANAUSKAS et al, 1998).

Essas referências ainda podem ser diretas ou indiretas como conceitua Familant e Detweiler 
(1993), na referência direta há somente um referente envolvido, denotativo, enquanto que na referência indireta há pelo menos dois referentes envolvidos, do signo e referente denotativo (BARANAUSKAS et al, 1998). A figura 3 exemplifica as referências diretas e indiretas.

Figura 3 - Referência direta e indireta.

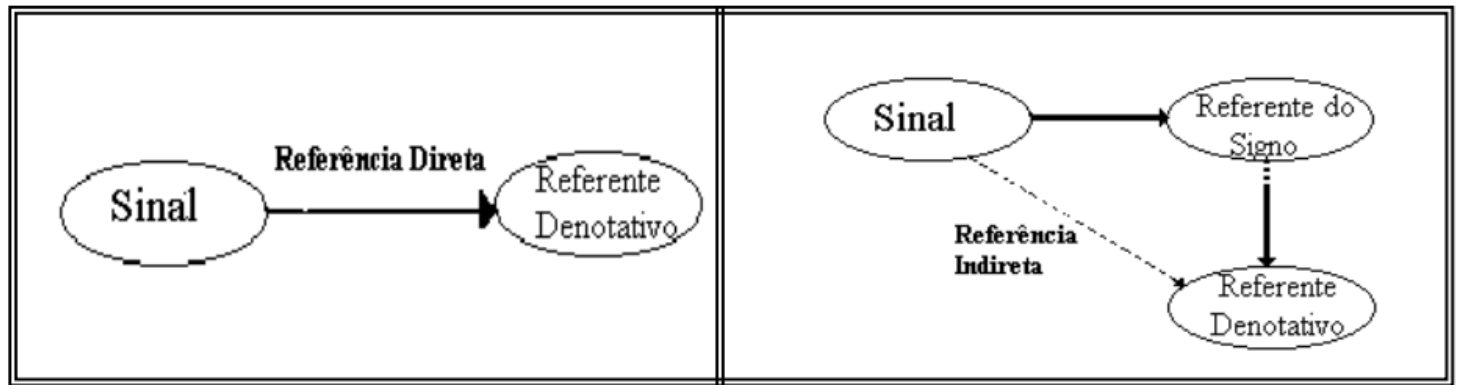

Fonte: Baranauskas et al (1998).

\section{Metodologia}

Para alcançar os objetivos deste trabalho primeiramente foi realizada uma revisão narrativa da literatura, que apresenta uma temática mais aberta não exigindo um protocolo rígido para sua execução (CORDEIRA et al, 2007), para os temas de inspeção de interface gráfica e semiótica. Após a revisão de literatura optou-se pelo estudo de caso, que se configura como uma investigação para compreender fenômeno contemporâneo em seu contexto, com coleta de dados quantitativos, qualitativos ou ambos (YIN, 2015). Para isto como metodologia utilizou-se a abordagem semiótica, constituída pelo framework apresentado na seção 3, referencial teórico, deste trabalho aplicado a plataforma MOOC TIMTec. A escolha da plataforma deu-se pelo critério de proximidade dos pesquisadores e a necessidade do maior conhecimento da mesma para sua utilização em projetos de extensão.

\section{Aplicação do Framework}

A aplicação dos conceitos de Andersen (1992) e Familant e Detweiler (1993) foi realizada na plataforma MOOC TIM Tec, apresentada na seção dois deste trabalho. O MOOC TIM Tec é uma plataforma educacional que oferece cursos gratuitamente, assim a interpretabilidade da IGU pelo usuário influencia no acesso ao material didático, moldando a experiência do usuário e o processo de aprendizado. Desta forma a IGU selecionada para a inspeção foi a interface de uma aula, figura 4. Para uma melhor visualização da aplicação da tipologia dos signos de Andersen (1992) a interface foi dividida em cinco figuras: Interface completa, menu principal, informações do curso, vídeo aula e player de vídeo. Para facilitar a interpretação das imagens cada tipo de signo foi numerado de acordo com o quadro 1.

Quadro 1 - Legenda de tipologia de signos.

\begin{tabular}{ll}
\hline Número & Tipo \\
\hline 1 & Interativo
\end{tabular}




$\begin{array}{ll}2 & \text { Ator } \\ 3 & \text { Objeto } \\ 4 & \text { Controlador } \\ 5 & \text { Fantasma } \\ 6 & \text { Layout } \\ 7 & \text { Botão }\end{array}$

Fonte: Desenvolvido pelo autor.

Ao todo foram identificados 20 signos interativos, 2 atores, 1 objeto, 1 controlador, 1 fantasma e 6 layouts. Na interface completa podemos perceber os signos do tipo Layout, sinalizados pelos números 6 na figura 4. Estes signos só possuem aspectos permanentes, não apresentam características de manuseio ou transientes, sendo apenas imagens decorativas para o fundo de tela.

Figura 4 - Interface completa MOOC TIM Tec.

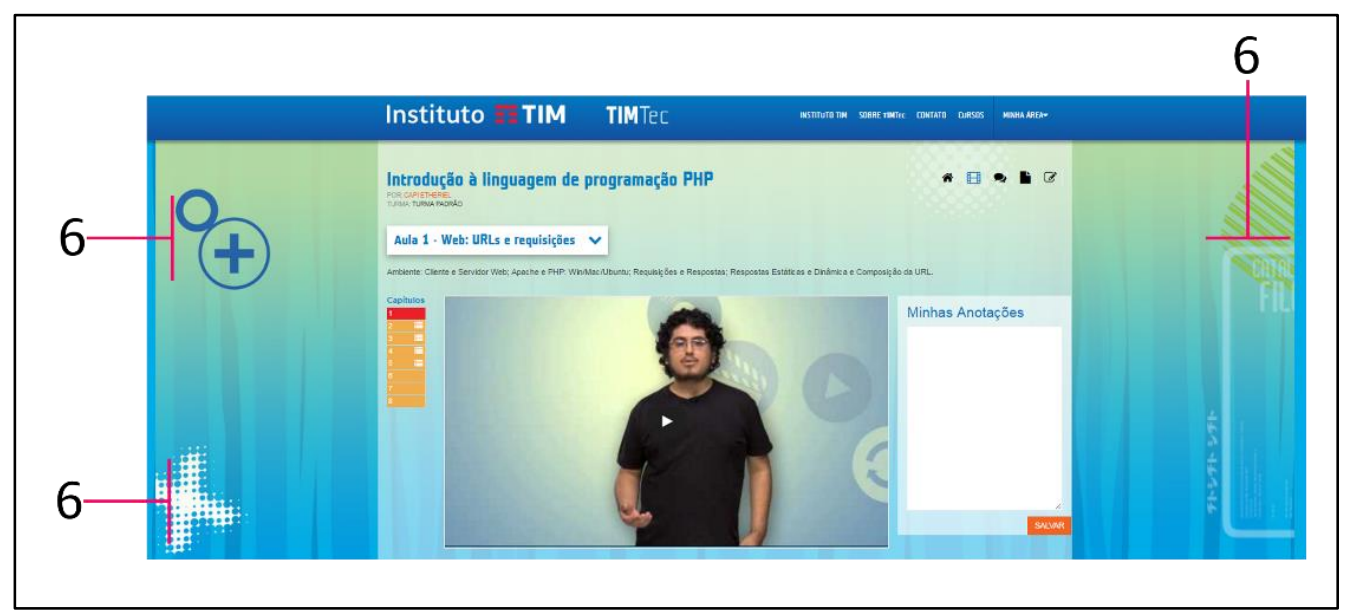

Fonte: Introdução à linguagem de programação PHP - TIM Tec (2018), adaptado pelo autor.

Na figura 5, menu principal do sistema, é possível perceber que todos os botões identificados pelo número 1 são do tipo Interativo, pois são manipulados diretamente pelo usuário e realizam uma ação em resposta.

Figura 5 - Menu principal MOOC TIM Tec.

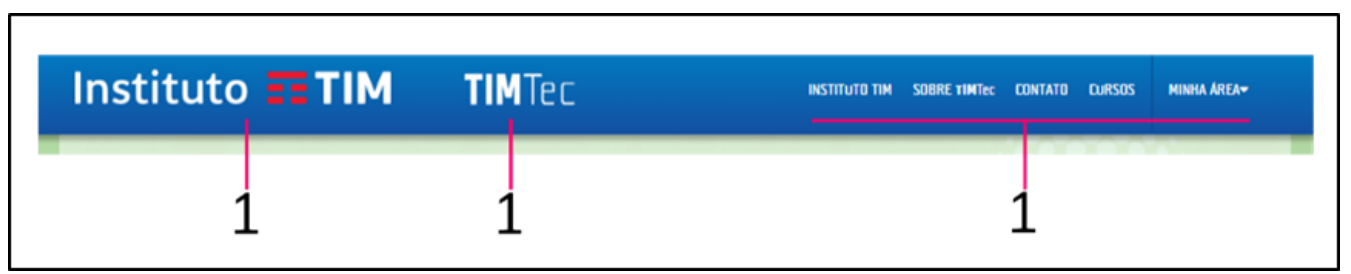

Fonte: Introdução à linguagem de programação PHP - TIM Tec (2018), adaptado pelo autor. 
Nas informações do curso, figura 6, há a predominância dos signos do tipo Interativo, e um signo de tipo Ator no texto de descrição da aula, que se altera de acordo com o capítulo selecionado, ação de outro signo e não ação direta do usuário.

Figura 6 - Informações do Curso MOOC TIM Tec.

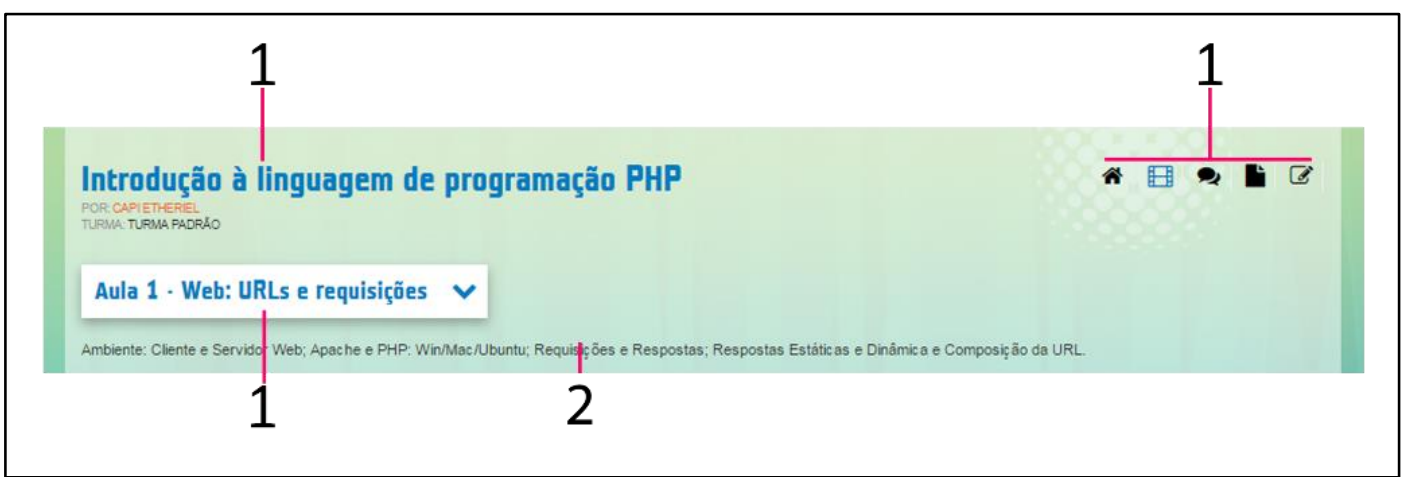

Fonte: Introdução à linguagem de programação PHP - TIM Tec (2018), adaptado pelo autor.

No vídeo aula do curso, figura 7, vemos além da presença de signos interativos, número 1, o signo número 3, objeto, que é um pequeno editor de texto, mas que não interage com outros signos. A borda do player de vídeo é um signo do tipo controlador pois com o passar do ponteiro do mouse muda de forma e altera a cor do botão de play do vídeo. Há também um signo fantasma, número 5 , na figura 7, que somente se torna visível quando o botão de play é pressionado, como mostra a figura 8.

Figura 7 - Vídeo aula MOOC TIM Tec.

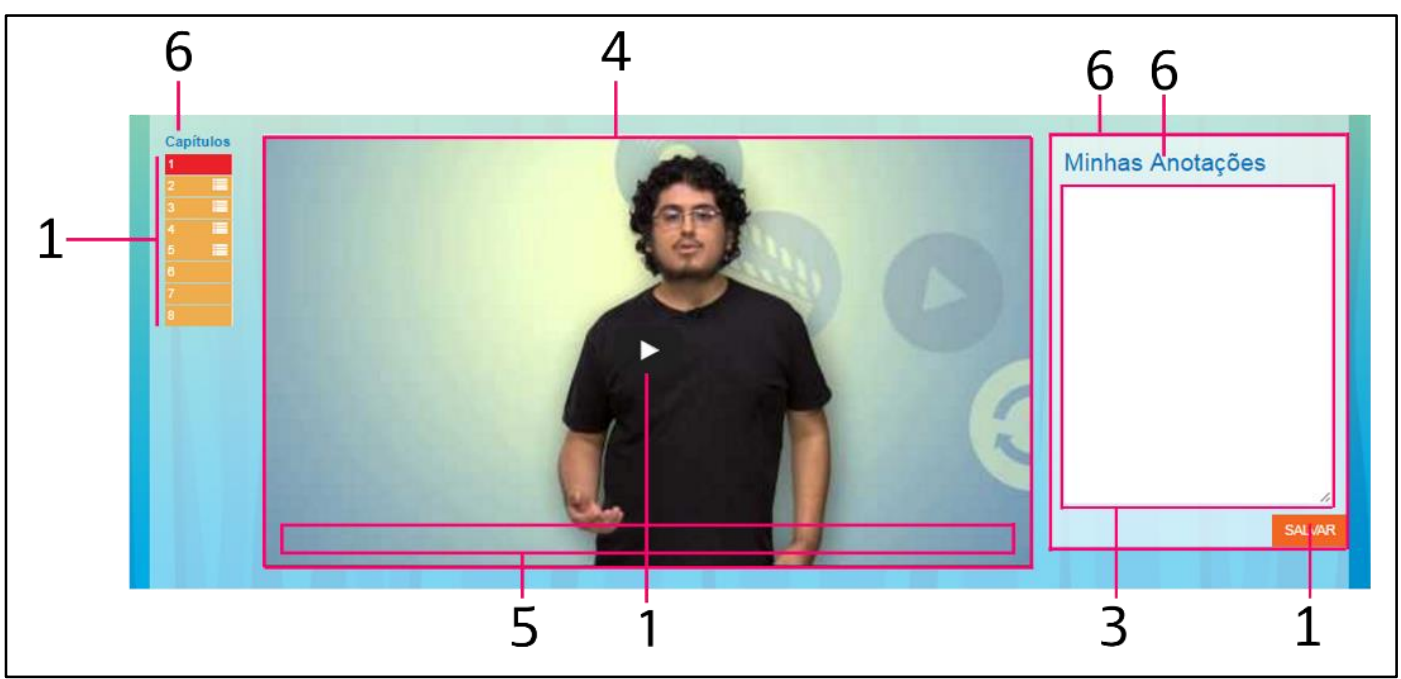

Fonte: Introdução à linguagem de programação PHP - TIM Tec (2018), adaptado pelo autor. 
Figura 8 - Player de vídeo MOOC TIM Tec.

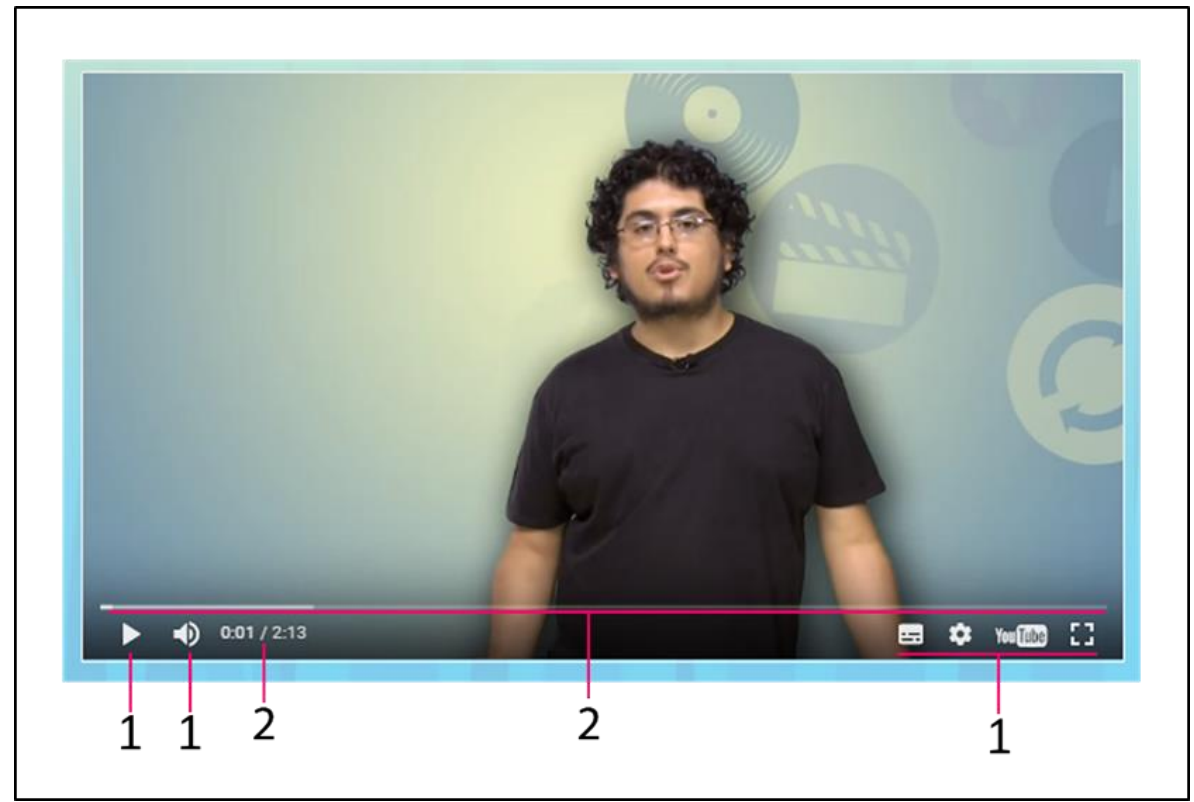

Fonte: Introdução à linguagem de programação PHP - TIM Tec (2018), adaptado pelo autor.

Para a interpretabilidade dos signos com o framework de Familant e Detweiler (1993), foi selecionado apenas o menu de opções do curso, visível no canto superior direito da interface, figura 6 , e em destaque na figura 9. Somente estes signos foram selecionados pois os demais apresentam signos puramente textuais, sempre levando a relações diretas.

Figura 9 - Menu opções do curso MOOC TIM Tec.

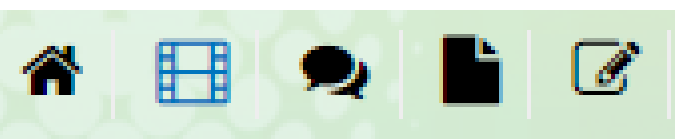

Fonte: Introdução à linguagem de programação PHP - TIM Tec (2018), adaptado pelo autor.

Como mostra o quadro 2, quatro dos cinco itens apresentam relação indireta e icônica. Nas relações icônicas o conjunto de propriedades do sinal tem elementos comuns ao conjunto de propriedades do referente, e nas simbólicas qualquer similaridade entre o sinal e seu referente é acidental, como acontece no primeiro signo da figura 10, uma casa indicando a página inicial do curso.

Quadro 2 - Propriedades de interpretabilidade dos botões MOOC TIM Tec.

\begin{tabular}{ll}
\hline Item & Interpretabilidade \\
\hline $\mathbf{A}$ & $\begin{array}{l}\text { Relação indireta, } \\
\text { simbólica }\end{array}$ \\
Relação indireta, \\
icônica
\end{tabular}




Relação indireta,
icônica

Fonte: Desenvolvido pelo autor.

O detalhamento do framework de Familant e Detweiler (1993) de dois itens do menu podem ser observados nas figuras 10 e 11. A figura 11 ilustra o conceito de relações indiretas simbólicas, onde relação do sinal casa e página inicial se relacionam de maneira indireta pois na língua inglesa ambas as palavras são escritas como home, sendo a interpretação para usuários não nativos desta língua uma associação indireta.

Figura 10 - Relação sígnica do botão página inicial.

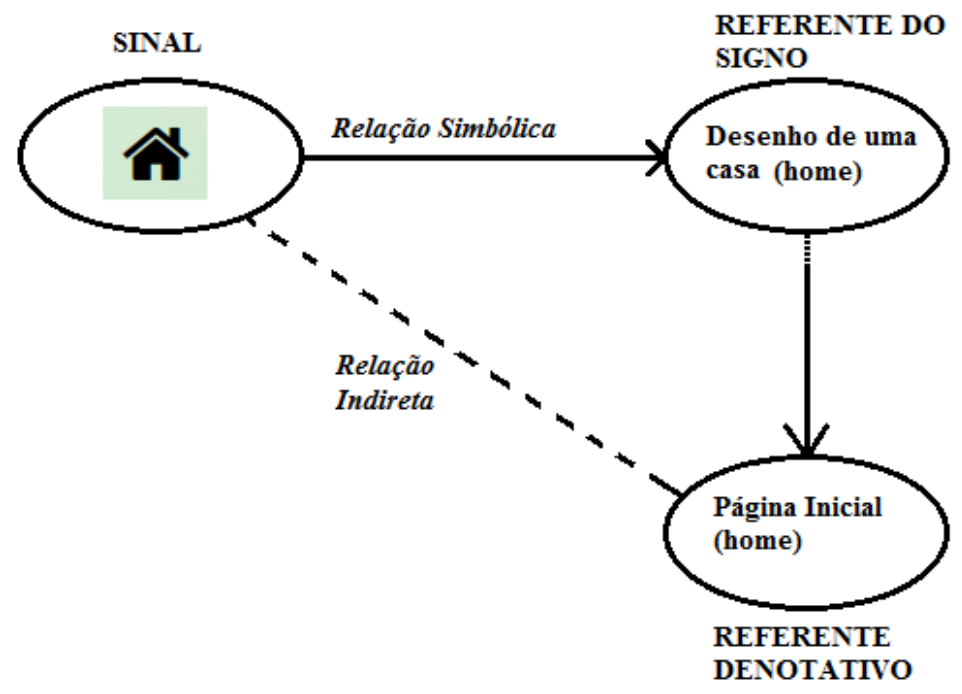

Fonte: Desenvolvido pelo autor.

Já na figura 11 é possível observar uma relação direta e icônica, onde o sinal e o seu referente denotativo representam um bloco de notas.

Figura 11 - Relação sígnica botão bloco de notas.

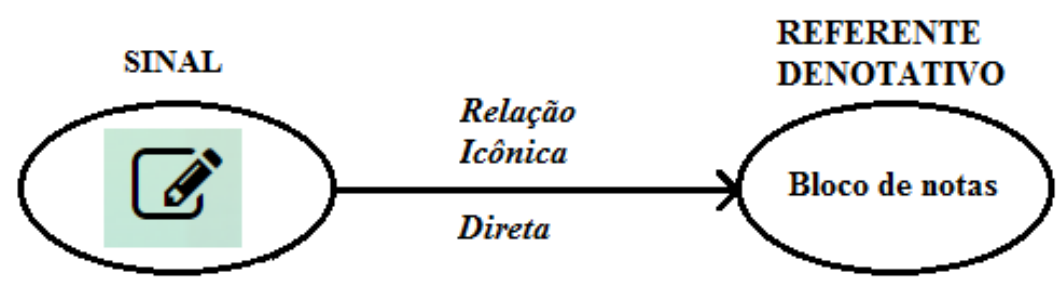

Fonte: Desenvolvido pelo autor. 


\section{Considerações Finais}

Sistemas computacionais, são sistemas baseados em signos que só possuem propósito quando estes são interpretados pelos usuários. No caso do MOOC TIM Tec a necessidade de ser plenamente interpretado por seu usuário está altamente ligado a qualidade da transferência de conhecimento oferecida pela plataforma. Para a semiótica computacional os desenvolvedores de software e designers de interface precisam estar atentos para alcançarem uma maior correspondência entre o sinal e seu referente, considerando os relacionamentos entre o conjunto de características do sinal e o conjunto de características do referente do signo, e também entre o conjunto de características do referente do signo e do referente denotativo (BARANAUSKAS et al, 1998).

$\mathrm{Na}$ inspeção de interface do MOOC TIM Tec foi encontrado uma alta concentração de signos interativos, o que apresenta um ótimo feedback para as ações dos usuários. Foi possível perceber que a grande parte dos signos eram compostos apenas de elementos textuais, que possuem relação direta com seu referente denotativo, sendo seus significados explícitos e de fácil interação com o usuário, porém podem ser menos atrativos ao usuário, pois interfaces com muitos textos podem ferir heurísticas de usabilidade, ocupando uma maior área gráfica da interface. Foi possível notar também que o plano de fundo de toda a interface página da vídeo aula é composto por signos do tipo layout. Este tipo de signo pode ser uma ótima maneira de tornar uma interface mais atrativa. Porém em excesso signos layout podem prejudicar a atenção do usuário, dificultar a leitura e tornar mais difíceis as interações com outros elementos da página.

A inspeção de interface com a tipologia de Andersen (1992) e o framework para interpretabilidade de Familant e Detweiler (1993) se mostraram adequados à inspeção de interface gráfica, apresentado bons resultados sobre a interpretabilidade dos signos. A avaliação com estes dois instrumentos é bastante subjetiva as interpretações do pesquisador, exigindo conhecimentos prévios tanto sobre o tipo de sistema usado e a sua conexão com o mundo real, assim como conhecimentos em computação e semiótica. Assim, a tipologia e o framework não esgotam o assunto ou são definitivos para se ter conhecimento preciso sobre a interpretabilidade para o usuário final, avaliações de usabilidade e de experiência do usuário por especialistas e testes com usuários podem ser muitos ricos para validar as informações deste estudo de caso e mesmo para propor melhorias a interface.

\section{Referências}

ANDERSEN, P. B. Computer Semiotics. University of Aarhus. Dinamarca. 1992

ANDERSEN, P B. A Theory of Computer Semiotics: Semiotic approaches to construction and assessment of computer systems. Cambridge University Press, 1997.

BARANAUSKAS, M. et al. Uma Abordagem Semiótica à Análise de Interfaces: um estudo de caso. São Paulo: IHC, 1998. p. 1-10.

CORDEIRO, A. et al. Revisão sistemática: uma revisão narrativa. 2007.

FACEBOOK. Facebook. Disponível em: <facebook.com>. Acesso em: 20 mar. 2018.

FAMILANT, M. Eliot.; DETWEILER, Mark. Iconic reference: evolving perspectives and an organizing 
framework. International Journal of Man-Machine Studies, V. 39, n. 4, p. 705-728. 1993.

INSTITUTO TIM. Instituto TIM. Disponível em: <https://institutotim.org.br/>. Acesso em: 6 fev. 2018.

INSTITUTO TIM. TIM Tec. Disponível em: <http://cursos.timtec.com.br/>. Acesso em: 06 fev. 2018.

PEIRCE, C. S. The Collected Papers of Charles Sanders Peirce. 1994.

NADIN, M. Interface design: A semiotic paradigm. Semiotica, v. 69, n. 3-4, p. 269-302, 1988.

OLIVEIRA, E.; LUZ, L.; PRATES, R. Aplicação semi-estruturada do método de inspeção semiótica: estudo de caso para o domínio educacional. In: Proceedings of the VIII Brazilian Symposium on Human Factors in Computing Systems. Sociedade Brasileira de Computação, 2008. p. 50-59.

SOCIEDADE BRASILEIRA DE COMPUTAÇÃO. Comissão Especial de Interação Humano-Computador. Disponível em: <http://comissoes.sbc.org.br/ce-ihc/index.php?content=apresentacao.txt>. Acesso em: 12 mar. 2018.

YIN, R. K. Estudo de Caso: Planejamento e Métodos. Bookman editora, 2015. 